\title{
A General Model of Population Dynamics Accounting for Multiple Kinds of Interaction
}

\author{
Luciano Stucchi, ${ }^{1,2}$ Juan Manuel Pastor, ${ }^{2}$ Javier García-Algarra $\mathbb{D}^{\mathrm{D}},{ }^{3}$ and Javier Galeano $\mathbb{i D}^{2}$ \\ ${ }^{1}$ Universidad Del Pacífico, Lima, Peru \\ ${ }^{2}$ Complex Systems Group, E. T. S. I. A. A. B, Universidad Politécnica de Madrid, Madrid, Spain \\ ${ }^{3}$ Department of Engineering, Centro Universitario U-TAD, Las Rozas, Spain \\ Correspondence should be addressed to Javier Galeano; javier.galeano@upm.es
}

Received 13 May 2020; Accepted 22 June 2020; Published 24 July 2020

Guest Editor: Tongqian Zhang

Copyright (c) 2020 Luciano Stucchi et al. This is an open access article distributed under the Creative Commons Attribution License, which permits unrestricted use, distribution, and reproduction in any medium, provided the original work is properly cited.

\begin{abstract}
Population dynamics has been modelled using differential equations almost since Malthus times, more than two centuries ago. Basic ingredients of population dynamics models are typically a growth rate, a saturation term in the form of Verhulst's logistic brake, and a functional response accounting for interspecific interactions. However, intraspecific interactions are not usually included in the equations. The simplest models use linear terms to represent a simple picture of the nature; meanwhile, to represent more complex landscapes, it is necessary to include more terms with a higher order or that are analytically more complex. The problem to use a simpler or more complex model depends on many factors: mathematical, ecological, or computational. To address it, here we discuss a new model based on a previous logistic-mutualistic model. We have generalized the interspecific terms (for antagonistic and competitive relationships), and we have also included new polynomial terms to explain any intraspecific interaction. We show that, by adding simple intraspecific terms, new free-equilibrium solutions appear driving a much richer dynamics. These new solutions could represent more realistic ecological landscapes by including a new higher order term.
\end{abstract}

\section{Introduction}

In the times of the coronavirus, many news on television and magazines try to explain how the size of the infected population evolves, showing exponential plots of the infected populations over time. These communications try to predict the time evolution of the size of this population in the future. Behind these predictions, there is always a differential equation model. These polynomial models have linear terms, but to account for more complex interactions, they can add higher order terms, as quadratic, cubic, or even, analytically more complex functions, such as decreasing hyperbolic terms. The problem of choosing a complex or a simple model depends on the balance between properly representing nature and being able to understand the model response. In many cases, the simplest model may be enough to understand the benchmarks in the big picture, but sometimes, we need more complexity to represent significant aspects of our problem, and therefore, we need more complex and more difficult models. Finding the balance between simple and complex is a tricky problem, but how simple or complex should the model be? Let us try to answer this question in a population dynamics problem.

In the study of population dynamics, Lotka [1] and Volterra [2] were the first ones to model trophic interactions in order to study predator-prey relationships within two (or more) populations:

$$
\begin{gathered}
\dot{X}_{1}=\left(r_{1}-b_{12} X_{2}\right) X_{1}, \\
\dot{X}_{2}=\left(-r_{2}+b_{21} X_{1}\right) X_{2},
\end{gathered}
$$

where $b_{i j}$ terms represent the rate of the interactions between populations $X_{i}$ and $X_{j}$ and the $r_{i}$ represents their effective growth rates. In these equations, signs are 
incorporated to give a clear meaning to each term, considering all parameters as positive real numbers. This simple model uses a linear term to represent the interaction with the environment and a pairwise second-order term to show the antagonistic interaction between the populations of two different species. It was necessary to introduce a higher order term, to represent this interaction.

Although most population dynamics models first dealt with antagonistic relations, mutualistic interactions are widely spread, e.g., [3, 4]. Garcia-Algarra et al. [5] proposed a logistic-mutualistic model. Their formulation was based on writing an effective growth rate as the sum of the intrinsic growth rate $\left(r_{i}\right)$ plus the mutualistic benefit $\left(b_{i j} X_{j}\right)$ and, associated with them, to include a saturation term for the whole effective growth term. The model was depicted as

$$
\begin{aligned}
& \dot{X}_{1}=\left(r_{1}+b_{12} X_{2}\right) X_{1}-\left(a_{1}+c_{1} b_{12} X_{2}\right) X_{1}^{2}, \\
& \dot{X}_{2}=\left(r_{2}+b_{21} X_{1}\right) X_{2}-\left(a_{2}+c_{2} b_{21} X_{1}\right) X_{2}^{2} .
\end{aligned}
$$

The term $a_{i} X_{i}^{2}$ represents the intraspecific competition for resources, and the term $c_{i} b_{i j} X_{j} X_{i}^{2}$ plays the role of saturation for the mutualistic benefit. This model needs to reach a thirdorder term to prevent the unbounded growth and depicts a well behaved system, with enough richness to model large ensembles of mutualistic networks and their behaviour.

Other authors have addressed different strategies to introduce the mutualistic interaction. For example, Dean [6] introduced an exponential dependency on the carrying capacity, $K$, which consequently yields nonlinear terms into the equations. To avoid the unbounded growth, the authors in $[7,8]$ proposed restrictions using a type II Holling functional response. This functional leaves the path of introducing a polynomial term with a hyperbolic function.

Nowadays, several studies have focused on adding higher order terms to explain more complex ecological interactions. Letten and Stouffer [9] studied the influence of interspecific interactions as nonadditive density-dependent terms only for competitive communities. Bairey et al. [10] studied the new solutions that a third species adds in pairwise interactions, adding third degree terms with the three different populations, $b_{i j k} X_{i} X_{j} X_{k}$. It is well known that an increase in the order of a polynomial term introduces new solutions to the equations, but as AlAdwani and Saavedra [11] showed these new terms do not always produce viable solutions, furthermore, they must be free-equilibrium points, and of course, the solutions must have an ecological meaning.

Here, we propose a new general model in which any ecological interaction can be included in a simple way. In a first step, we generalise the model proposed by GarciaAlgarra et al. [5] overcoming the restrictions of the sign of the parameters; in a second step, we reorganize the intraspecific interactions allowing for both positive and negative interactions, and finally, we introduce a third-order term to brake any unbounded pairwise interactions.

\section{Methods and Materials}

We define a new general model. Equation (3) represents the population dynamics of the species $X_{i}$ driven by an effective growth rate (first parenthesis in equation (3)) and limited by a logistic brake (second parenthesis in equation (3)). The view of the model is simple and similar to the original Verhulst idea [12], where the low-order terms represent the increase in the population and the high-order terms the brake. The differences with other models are in the terms included in the effective growth rate and logistic brake. The effective growth rate includes the vegetative growth rate, $r_{i}$, and all density-dependent pairwise interactions, interspecific interactions, $b_{i j} X_{j}(\forall j \neq i)$, and intraspecific ones, $b_{i i} X_{i}$; the logistic brake includes the logistic term due to intraspecific competition, $a_{i}$, the interspecific intraspecific brake, $b_{i j} X_{j} X_{i}$, and the intraspecific ones, $b_{i i} X_{i}^{2}$.

2.1. A New General Model including Intraspecific Interaction Terms. Regarding the mutualistic model (equation (2)) we introduce two differences: first, the parameters of the equation, $r_{i}$ and $b_{i j}$, can be positive or negative, representing the different ecological interactions, and second, we include the effect of the population in its own effective growth rate just adding the index $j=i$ in the sum of the interactions terms, so the model can be represented as

$$
\dot{X}_{i}=X_{i}\left[\left(r_{i}+\sum_{j=1}^{n} b_{i j} X_{j}\right)-\left(a_{i}+c_{i} \sum_{j=1}^{n} b_{i j} X_{j}\right) X_{i}\right] \text {, }
$$

where the subscript $i$ runs from 1 to $n$, including the intraspecific interaction $(j=i)$. With this term, we are taking into account the interaction between individuals of the same populations. The new terms yield new solutions and a different phase space. In particular, the inclusion of the term $-c_{i} b_{i i} X_{i}^{3}$ is key for the emergence of new solutions although there was already a term with the same order in the mutualistic logistic model (equation (2)), $c_{i} b_{i j} X_{j} X_{i}^{2}$. It can be observed in Figure 13 in Supplementary Materials (available here). We explain all details about the number of solutions in Appendix in Supplementary Materials (available here).

Generally, in the literature of populations dynamics, the intraspecific interactions have been introduced only as a logistic brake, $-a_{i} X_{i}$, representing a growth limit due to resource sharing. In our model, the term, $b_{i i}$, can represent any kind of intraspecific interaction from beneficial, namely, cooperation to harmful interactions, such as competition or even cannibalism. Even though the logistic term $-a_{i} X_{i}$ can be seen as the result of intraspecific interactions that limit the growth by resource sharing and it can be included in the interaction term $b_{i i} X_{i}$, we maintain the separated formulation for the sake of comparison with the equation without this new term.

In fact, there are abundant examples of different intraspecific behaviours in the literature, such as those mentioned above. Cooperation is well known among social and eusocial species [13], and benefits of cooperative behaviour have been consistently reported, especially for eusocial animals [14]. On the other way, in nature, we can find different types of competition among members of the same population. For example, Stucchi and Figueroa [15] reported the aggressive intraspecific behaviour of the 
Peruvian booby, which attacks their peers not by means of taking their food but for the sake of being around their nest. In the same way, adult boobies show little tolerance for pigeons that are not from them, pecking them to death. This behaviour is well known for other territorial animals, and it conceptually differs from the conventional intraspecific competition for resources.

2.2. Solutions with One Population. In general, the equation system (3) cannot be solved analytically. However, the study of only one population can be solved and illustrates the possibilities of the model.

Consider equation (3) for only one population. The equation can be written as

$$
\dot{X}=X[(r+b \cdot X)-(a+c b \cdot X) X],
$$

where we have removed the subscripts for simplicity. Stationary points where $\dot{X}=0$ give us the keys to understand the behaviour of the time evolution of the population sizes. The trivial solution, which corresponds to extinction, is $X^{\star}=0$. Now, the nontrivial stationary solutions can be obtained from the following condition:

$$
r+(b-a) X^{\star}-c \cdot b X^{\star 2}=0 .
$$

Then, the stationary solutions of equation (4) are the extinction and the solutions of equation (5):

$$
X_{ \pm}^{*}=\frac{(b-a) \pm \sqrt{(b-a)^{2}+4 r b c}}{2 b c} .
$$

In ecology, we are only interested in the positive real solutions, generally called feasible solutions. To obtain these feasible solutions in equation (6), we need to study several cases:

(i) $r$ and $b$ have the same sign. In addition to the trivial solution, $X^{\star}=0$, in both cases, there is one positive stationary point, which corresponds to the carrying capacity of the population, and the other is negative, which is not a feasible solution.

(a) In the case that both parameters are negatives, $r<0$ and $b<0$, the positive solution is unstable and the trivial solution is the unique stable solution.

(b) In the opposite case, $r>0$ and $b>0$, the carrying capacity is the stable solution.

(ii) $r$ and $b$ have different signs. The interesting point of having a high-order term comes from the possibility of different signs of the parameters. When $r$ and $b$ have different signs, there are two solutions as long as the condition $c \leq(b-a)^{2} / 4|r b|$ is fulfilled.

(a) If $b>0$, it is a necessary another condition to obtain a feasible solution that $b>a$. Ecologically speaking, this means that the term of intraspecific interaction overcomes the intrinsic growth deficiency and increases the population. In Figure 1(b), we plot a case with these conditions. We obtain three fixed points: initial and end points are stable, and the intermediate point is unstable. This point marks the threshold population; above this value, intraspecific cooperation moves the population to reach the carrying capacity, and below this value, the population goes to extinction.

(b) If $b<0$. In this scenario, the intermediate point is stable, and the other solutions are unstable. Consequently, the intraspecific competitions produce a new stationary solution, lower than the carrying capacity. This behaviour has been called as Allee effect [16]. See the example in Figure 1(b).

In Figure 1, we depict on the top $\dot{X}$ vs. $X$ and on the bottom, the temporal evolution of the population size $X(t)$ vs. $t$. On the left, the growth rate $r$ is negative, and the intraspecific interaction coefficient $b$ is positive. The intermediate stationary solution plays the role of a population threshold because smaller communities will go extinct (population in orange in Figure 1(c)), while larger communities will grow to its carrying capacity (population in green in Figure 1(c)). On the right, the growth rate $r$ is positive, and the interaction coefficient $b$ is negative. In this case, the carrying capacity becomes unstable, and the system evolves to the new stable intermediate solution because of the detrimental intraspecific interaction (both populations in Figure 1(d)). Two examples of population evolution are plotted in each scenario, where the orange and green dots in the upper plot depict the initial condition of each evolution in the lower plot.

2.3. Solutions with Two Populations. In the case of two populations, the general model is written as

$$
\begin{aligned}
& \dot{X}_{1}=X_{1}\left[\left(r_{1}+b_{11} X_{1}+b_{12} X_{2}\right)-\left(a_{1}+c_{1} b_{11} X_{1}+c_{1} b_{12} X_{2}\right) X_{1}\right] \\
& \dot{X}_{2}=X_{2}\left[\left(r_{2}+b_{22} X_{2}+b_{21} X_{1}\right)-\left(a_{2}+c_{2} b_{22} X_{2}+c_{2} b_{21} X_{1}\right) X_{2}\right] .
\end{aligned}
$$

For two populations, we also find the expected trivial solution, i.e., the total extinction $\left(X_{1}^{\star}=0\right.$ and $\left.X_{2}^{\star}=0\right)$ and the partial extinctions $\left(X_{1}^{\star}, 0\right)$ and $\left(0, X_{2}^{\star}\right)$, from the following equations:

$$
\begin{aligned}
& r_{1}+\left(b_{11}-a_{1}\right) X_{1}^{\star}-c_{1} b_{11} X_{1}^{\star 2}=0, \\
& r_{2}+\left(b_{22}-a_{2}\right) X_{2}^{\star}-c_{2} b_{22} X_{2}^{\star 2}=0 .
\end{aligned}
$$

However, as they are second-order equations, there are two solutions of feasible partial extinctions for each population. The coexistence solutions can be obtained from equation (7); these equations can exhibit up to 6 new stationary solutions. Concerning the finite stationary solutions, the intraspecific term makes it more difficult to obtain an analytic expression from the following equations:

$$
\begin{aligned}
& \left(r_{1}+b_{11} X_{1}^{\star}+b_{12} X_{2}^{\star}\right)-\left(a_{1}+c_{1} b_{11} X_{1}^{\star}+c_{1} b_{12} X_{2}^{\star}\right) X_{1}^{\star}=0, \\
& \left(r_{2}+b_{22} X_{2}^{\star}+b_{21} X_{1}^{\star}\right)-\left(a_{2}+c_{2} b_{22} X_{2}^{\star}+c_{2} b_{21} X_{1}^{\star}\right) X_{2}^{\star}=0 .
\end{aligned}
$$




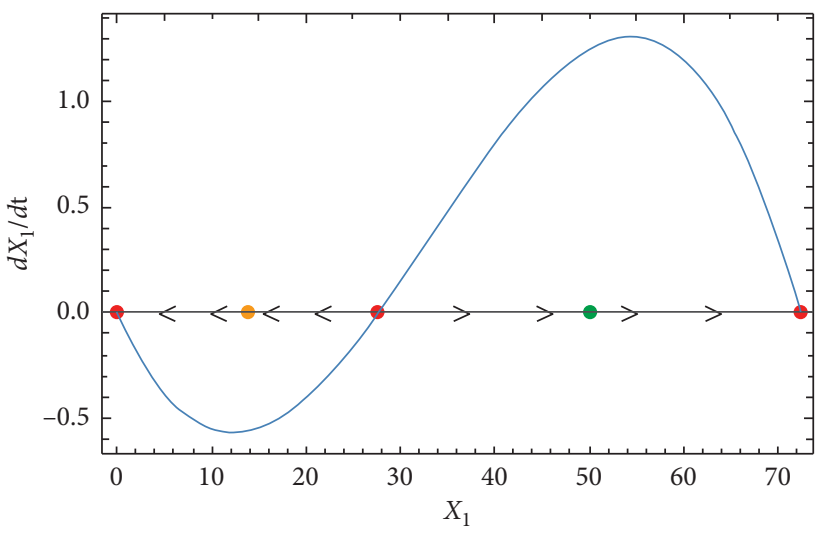

(a)

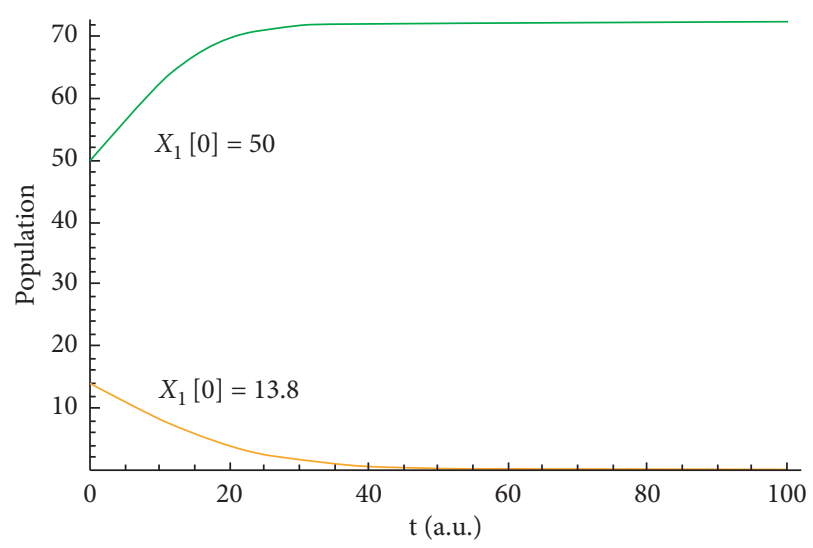

(c)

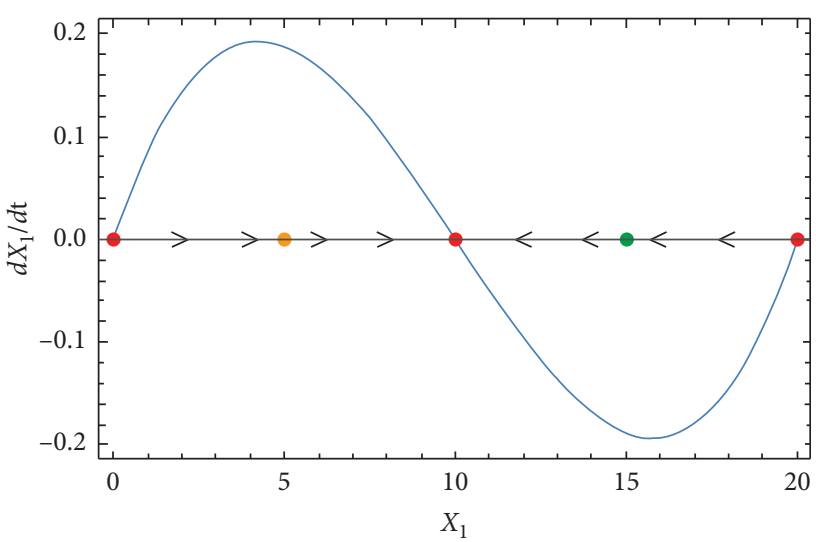

(b)

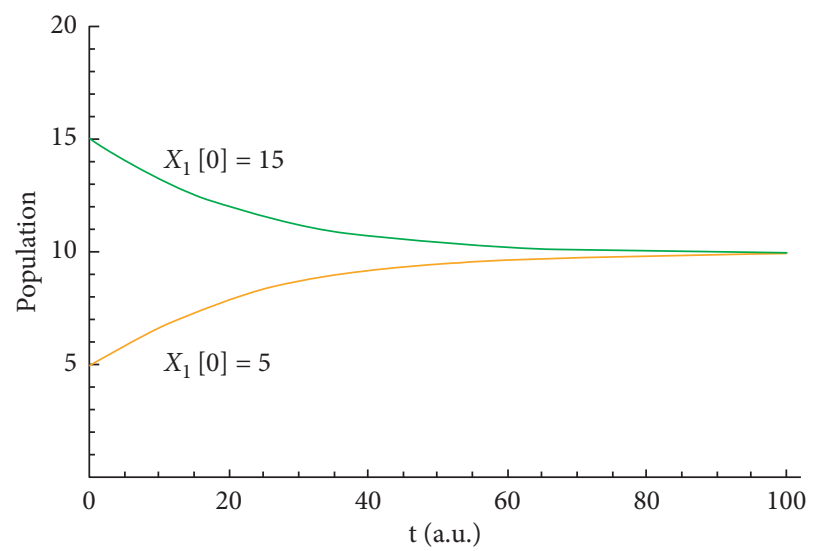

(d)

Figure 1: Temporal derivative (up) and population evolution (down) for one population with intraspecific interaction. Negative growth rate (left), $r=-0.1$, with positive intraspecific interaction $b=0.005$ and $c=0.005$. Positive growth rate (right), $r=0.1$, with negative intraspecific interaction $b=-0.015$ and $c=0.05$.

Two out of these six solutions are new free-equilibrium points, due to the new intraspecific terms (details in Supplementary Material). Even though we cannot obtain analytic expressions for all solutions, we explored different scenarios by performing numerical simulations with different parameter values. In the next section, we show how the intraspecific interaction changes the phase space of the standard biological interactions.

2.4. Linear Stability Analysis. In the next section, we explore the linear stability analysis of our system solutions.

2.4.1. One Population Model. To perform the linear stability analysis of the stationary solutions, we derive equation (4) at the fixed points:

$$
\left.\frac{\mathrm{d} \dot{X}}{\mathrm{~d} X}\right|_{X=0}=r
$$

$$
\left.\frac{\mathrm{d} \dot{X}}{\mathrm{~d} X}\right|_{X=X_{ \pm}^{\star}}=r+\left[2(b-a)-3 c b X^{\star}\right] X^{\star}=-r-c b X^{\star 2} .
$$

In the trivial solution, the eigenvalue is $\lambda=r$ and the unique stable solution is $r<0$.

According to equation (11), the derivative at the (positive) stationary solution $X^{\star}$ will be negative when

$$
(b-a)-2 c b X_{ \pm}^{\star}=\mp \delta<0 .
$$

Then, $X_{+}^{\star}$ is always stable and $X_{-}^{\star}$ is unstable.

When $r>0$ and $b>0$, extinction is an unstable solution and population rises to the carrying capacity at $X_{+}^{\star}$, the only positive nontrivial solution. However, for $r>0$ and $b<0$, i.e., with intraspecific competition, a new stationary solution emerges, $X_{-}^{\star}>X_{+}^{\star}$. Now, the higher solution is unstable, and the population only reaches a lower value at the stable point $X_{+}^{\star}$. In this case, the negative intraspecific interaction results in a lower carrying capacity.

When $r<0$, extinction is stable. If $b<0$, the only positive finite solution is $X_{-}^{\star}$, which is unstable. However, when $b>a>0$, a new stable solution, $X_{+}^{*}$, emerges at higher values than $X_{-}^{\star}$. In this scenario, $X_{-}^{\star}$ marks the threshold population; above this value, intraspecific cooperation moves the population to reach the carrying capacity, and below this value, the population goes to extinction (see Figure 1(c)). 
2.4.2. Two Populations Model. The linear stability for the general model (equation (3)) can be analyzed from the Jacobian matrix at the stationary solutions. Its entries are obtained from

$$
\left.\frac{\partial f_{i}}{\partial X_{i}}\right|_{X^{\star}}=g_{i}\left(X^{\star}\right)+\left(b_{i i}-a_{i}\right) X_{i}^{\star}-2 c_{i} b_{i i} X_{i}^{\star 2}-c_{i} \sum_{j \neq i} b_{i j} X_{i}^{\star} X_{j}^{\star} \text {, }
$$$$
\left.\frac{\partial f_{i}}{\partial X_{j}}\right|_{X^{\star}}=b_{i j} X_{i}^{\star}\left(1-c_{i} X_{i}^{\star}\right)
$$

where $X^{\star}=\left(X_{1}^{\star}, \ldots, X_{i}^{\star}, \ldots, X_{j}^{\star}, \ldots\right)$ is the vector of the stationary solution.

For two populations, the Jacobian matrix for the total extinction is

$$
J_{\{0,0\}}=\left(\begin{array}{cc}
r_{1} & 0 \\
0 & r_{2}
\end{array}\right),
$$

whose eigenvalues $\lambda_{1}=r_{1}$ and $\lambda_{2}=r_{2}$ are negative when both growth rates are negative. For the partial extinctions, the Jacobian matrix reads

$$
J_{\left\{X_{1}^{\star}, 0\right\}}=\left(\begin{array}{cc}
-r_{1}-c_{1} b_{11} X_{1}^{\star^{2}} & b_{12} X_{1}^{\star}\left[1-c_{1} X_{1}^{\star}\right] \\
0 & r_{2}+b_{21} X_{1}^{\star}
\end{array}\right) .
$$

As expected, this Jacobian matrix is almost the same as the matrix for the logistic-mutualistic model (see Appendix $A$ in [5]), but the first entry includes the intraspecific interaction term $-c_{1} b_{11} X_{1}^{\star 2}$. This new term makes the partial extinction to be stable when the intraspecific interaction is positive $b_{11}>0$. The same is stated for the symmetric solution $\left(0, X_{2}^{\star}\right)$.

And for the nontrivial solution $\left(X_{1}^{\star}, X_{2}^{\star}\right)$, the Jacobian matrix is written as follows:

$$
J_{\left\{X_{1}^{\star}, X_{2}^{\star}\right\}}=\left(\begin{array}{cc}
-r_{1}-b_{12} X_{2}^{\star}-c_{1} b_{11} X_{1}^{\star^{2}} & b_{12} X_{1}^{\star}\left[1-c_{1} X_{1}^{\star}\right] \\
b_{21} X_{2}^{\star}\left[1-c_{2} X_{2}^{\star}\right] & -r_{2}-b_{21} X_{1}^{\star}-c_{2} b_{22} X_{2}^{\star^{2}}
\end{array}\right) .
$$

In this case, both diagonal entries include the intraspecific term with a negative sign. This means that a positive intraspecific direct interaction enhances the stability of this stationary solution, while a negative intraspecific direct interaction contributes to destabilize it.

A qualitative study of the linear stability can also be made by analyzing the nullclines. Solving the nullclines, $f_{1}\left(X_{1}, X_{2}\right)=0$, we obtain two solutions $X_{1}=0$, as follows:

$$
\begin{aligned}
g_{1}\left(X_{1} \neq 0\right)= & r_{1}+\left(b_{11}-a_{1}\right) X_{1}-c_{1} b_{11} X_{1}{ }^{2} \\
& -\left(c_{1} X_{1}-1\right) b_{12} X_{2}=0,
\end{aligned}
$$

or writing $X_{2}$ in terms of $X_{1}$ :

$$
X_{2}\left(g_{1}=0\right)=\frac{r_{1}+\left(b_{11}-a_{1}\right) X_{1}-c_{1} b_{11} X_{1}^{2}}{b_{12}\left(c_{1} X_{1}-1\right)} .
$$

This expression presents a discontinuity at $X_{1}=1 / c_{1}$ and at $X_{2}=1 / c_{2}$ for the $f_{2}$ nullcline. At this discontinuity, the growth rate of species 1 takes the value,
$g_{1}\left(X_{1}=1 / c_{1}\right)=r_{1}-a_{1} / c_{1}$, independently of $X_{2}$ (and the same for $\left.g_{2}\left(X_{2}=1 / c_{2}\right)\right)$. The condition for a bounded growth leads to $c_{1} \leq a_{1} / r_{1}$, and as in Verhulst's equation, this parameter, $1 / c_{1}$, plays the role of the carrying capacity. With the same condition for species 2, i.e., $c_{2} \leq a_{2} / r_{2}$, we may define a rectangle limited by $X_{1}=0, X_{1}=1 / c_{1}, X_{2}=0$, and $X_{2}=1 / c_{2}$ in whose boundary the flux vectors never point out of the rectangle, and therefore, the growth is bounded.

Figure 2 depicts the bounding rectangle limited by the axis and the dashed lines $1 / c_{1}$ and $1 / c_{2}$. In Figure $2(a)$, the conditions $c_{1} \leq a_{1} / r_{1}$ and $c_{2} \leq a_{2} / r_{2}$ are fulfilled, and the flux lines are pointing inside the rectangle. In Figure 2(b), the conditions are no longer satisfied, but one stable solution is located outside the rectangle, allowing some flux lines to go out. The asymptotic behaviour of the nullcline at $X_{1}=1 / c_{1}$ has changed and now it rises to infinity.

The intersection of both nullclines defines the stationary solutions. As the expression equation (18) is nonlinear, there can be several solutions inside the rectangle. This allows more than one stable solution inside this area, separated by saddle points. As an example, Figure 2 shows the intersections of nullclines (black lines for $X_{1}$ and orange lines for $X_{2}$ ) as red points; two of them are stable stationary solutions, separated by a saddle point. In this example for a predator-prey system, the phase space shows the typical solution of a stable spiral (at $X_{1}=42$ and $X_{2}=79$ ) and a new stable node at a higher population of predator and prey (at $X_{1}=200$ and $X_{2}=164$ ). Note that even though $a_{1}$ does not fulfil the condition $a_{1} \leq c_{1} \cdot r_{1}$, in this example, the system is also bounded and stable outside the rectangle. Finally, the same study can be done for $N$ species. For every species, the value $X_{i}=1 / c_{i}$ can define a threshold for the initial population for which the flux trajectories never go outside the $\mathrm{N}$-dimensional rectangle. In this case, freeequilibrium solutions will be harder to obtain; however, the Jacobian at these points will have a similar expression (see Supplementary Material).

2.5. Solutions with Three Populations. Ecological complexity increases with species number. Just as a little example, we show in this section how the intraspecific interaction can change the outcomes in a 3-species predator-prey system. We show how a positive coefficient in the intraspecific term of the prey-1 avoids the extinction. Figure 3(a) shows the time evolution of three populations: two preys and one predator; the cooperation coefficient in prey-1 $\left(b_{11}=0.001\right)$, even smaller than the interspecific coefficient $\left(b_{13}=-0.004\right)$, changes the initial outcome resulting in a stationary population for prey-1 and predator and the extinction of prey-2.

For the case of negative intraspecific interaction, we show another predator-prey system, with two preys and one predator. In this example, the intraspecific coefficient of the predator $\left(b_{33}=-0.0005\right)$ allows both preys to survive at higher populations (Figure $3(\mathrm{~b})$ ); the three populations exhibit initial oscillations until they reach a stationary population; however, the difference in the interspecific coefficient $\left(b_{13}=-0.004\right.$ and $\left.b_{23}=-00045\right)$ makes the 


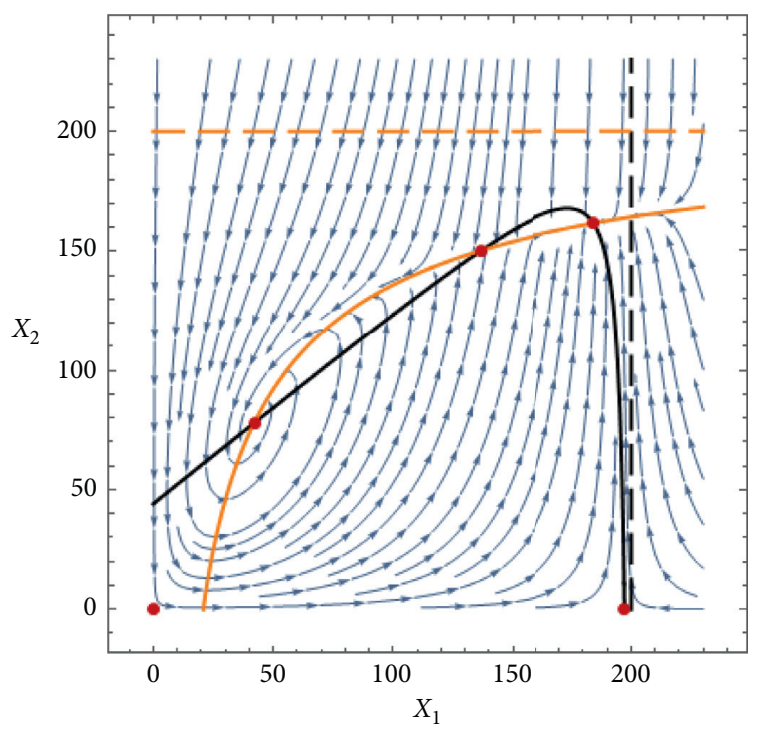

(a)

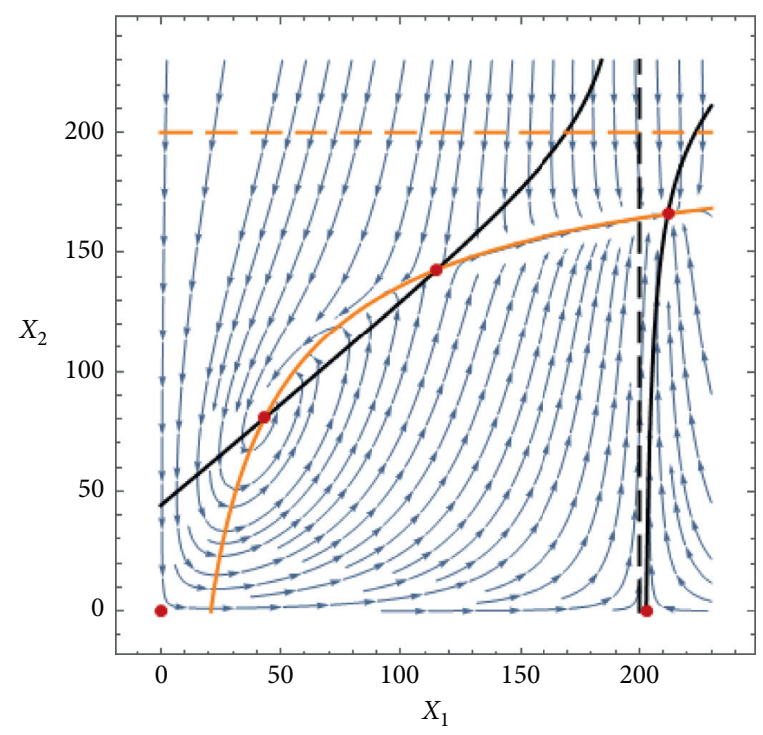

(b)

FIGURE 2: Nullclines and phase space for an antagonistic system where both populations cooperate intraspecifically. Dashed lines represent $X_{i}=1 / c_{i}$, and while solid lines are the nullclines (orange for $X_{2}$ and black for $X_{1}$ ). (a) $a_{1}=0.0008$ fulfils the condition $a_{1}>c_{1} \cdot r_{1}$, and flux lines inside the rectangle do not point out of this region. (b) $a_{1}=0.0007<c_{1} \cdot r_{1}$, and some flux lines go out of the rectangle. Parameters: $r_{1}=0.15, r_{2}=-0.15, b_{11}=0.0028, b_{12}=-0.0034, b_{21}=0.0072, b_{22}=0.0005, a_{2}=0.00075$, and $c_{1}=c_{2}=0.005$.

prey-1 stationary population to be higher than that of prey-2.

\section{Results}

Here, we show the great variety of scenarios of ecological interactions that this general model is capable of producing. The aim of this section is to show the great richness of the model, but it is not an exhaustive study of the parameters. We show some examples of the solutions that the intraspecific interaction provides to the populations model with two populations. Since exploring all the possible combinations of signs and ratios among the parameters would be unmanageable and redundant, we only show some interesting cases. For all the figures shown in this section, we have varied the parameters in the effective growth rate, $r_{i}$, $b_{i i}$, and $b_{i j}$, and we have set the limiting parameters $a_{1}=$ $a_{2}=0.00075$ and $c_{1}=c_{2}=0.005$.

3.1. Antagonism. The solutions of the classical predator-prey model are modified when intraspecific interactions come into play. In our following examples, we have $X_{1}$ as the prey and $X_{2}$ as the predators. We only show obligate predation since the facultative case only offers a minor change.

3.1.1. The Effect of Cooperation among Prey. The predatorprey system without any intraspecific interaction has only two free-equilibrium solutions: one convergent spiral and one unstable solution, located at the carrying capacity of the prey. The addition of cooperation among the population of preys can generate a new stable solution. Besides the well known oscillatory solution, we may find a new stable node at high population values, separated by a saddle point. Figure 4(a) shows the phase space and trajectories (with the stationary solutions as red points) for a predator-prey system when preys $\left(X_{1}\right)$ exhibit positive intraspecific interaction (cooperation). Phase trajectories keep around the stable spiral for low populations; however, the saddle point defines a new basin towards the new stable solution for high population values (note that the intraspecific parameter, $b_{11}=0.0028$, is lower than the absolute value of the interspecific parameter, $\left.b_{12}=-0.0036\right)$.

If the detrimental interspecific interaction becomes less harmful, the original stable spiral may disappear and the only stable solution is the coexistence at the carrying capacity (Figure $4(\mathrm{~b})$, with $b_{12}=-0.0036$ ).

When the intraspecific interaction is greater than the interspecific interactions, in our example, $\left|b_{12}\right|=b_{21}<b_{11}$, a new dynamic appears. The spiral becomes unstable, and the trajectories go outwards; as this stationary solution is in the repulsion basin of the saddle point, the trajectories cannot go out, and they will remain in a closed orbit, i.e., in a limit cycle. In Figure 5(a) (with $b_{11}=0.0036$ and $b_{12}=-0.0072$, in addition to representing the trajectories and the stationary solutions), we depict 3 initial points (in green, yellow, and orange) corresponding to the time evolution picture shown below. The intermediate solution that appeared due to the cooperation term acts as a threshold between the spiral and the coexistence located at the carrying capacity of the prey, which remains as a stable solution. Now, if we decrease the intraspecific parameter, the saddle point moves towards the carrying capacity, all the stationary solutions become unstable, and all the trajectories fall into the limit cycle 


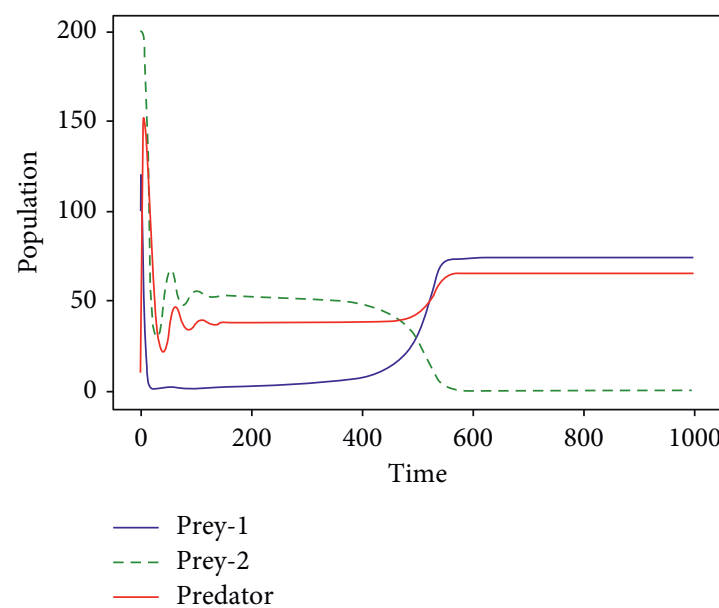

(a)

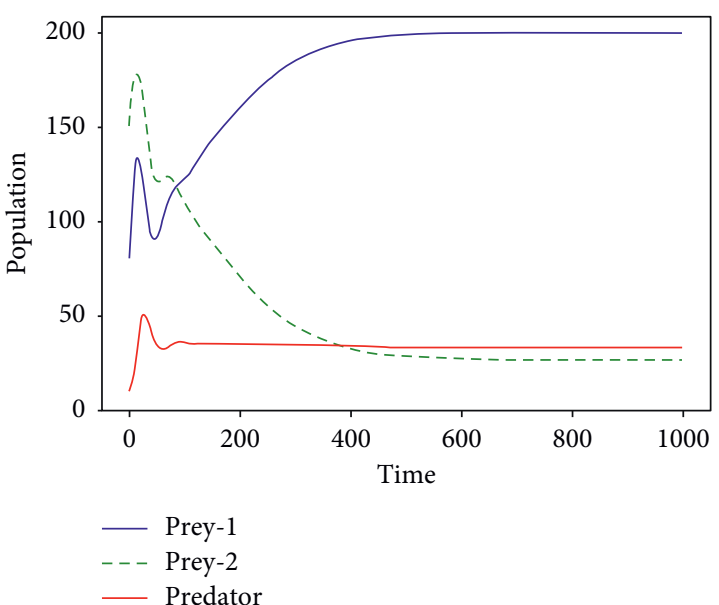

(b)

Figure 3: Population evolution in a predator-prey system with two preys: (a) prey-1 with cooperation $b_{11}=0.001\left(r_{1}=r_{2}=0.15\right.$, $r_{3}=-0.15, b_{13}=b_{23}=-0.004$, and $\left.b_{31}=b_{32}=0.004\right)$; (b) predator with competition $b_{33}=-0.0005 \quad\left(r_{1}=r_{2}=0.15, r_{3}=-0.15\right.$; $b_{13}=-0.004, b_{23}=-0.0045$, and $\left.b_{31}=b_{32}=0.001\right)$.

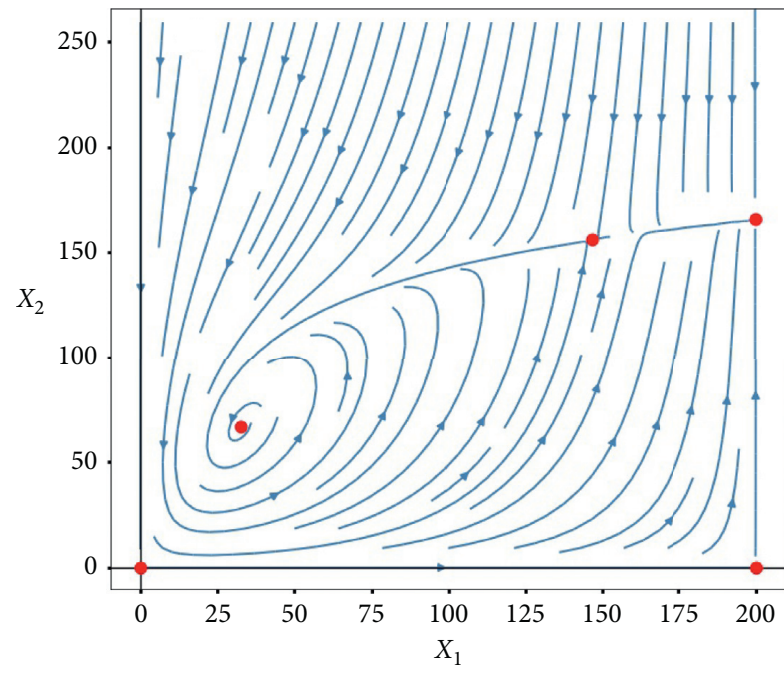

(a)

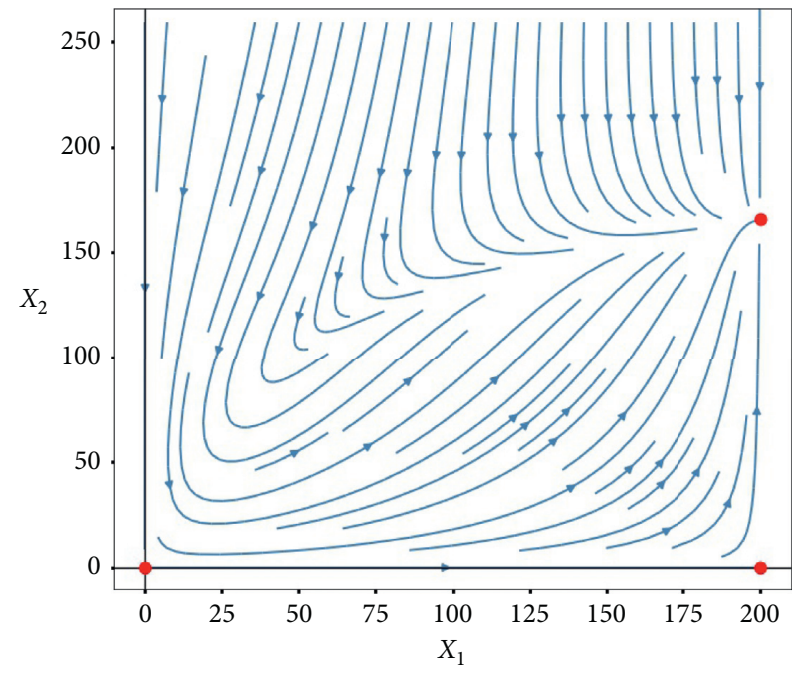

(b)

Figure 4: Phase space and trajectories for two populations involved in a predator-prey interaction. To the left, we have a case with a lower $b_{11}=0.0028$ than to the right, $b_{11}=0.0035$. Cooperation among prey allows a new intermediate solution, which is unstable and acts in the same way as in Figure 1. Also, as greater cooperation decreases the predatory term, and the relation may become commensalistic at some points. Here, $r_{1}=0.15, r_{2}=-0.15, b_{12}=-0.0036$, and $b_{21}=0.0072$.

(Figure 5(b)). The corresponding time evolution (Figure 5(b)) shows fluctuating population for all initial points.

3.1.2. The Effect of Cooperation among Predators. In Figure 6, we show the effect of the intraspecific interactions only on predators. As in the previous case, without any intraspecific interaction, the system has only two free-equilibrium points: one convergent spiral and one unstable solution, located at the carrying capacity of the prey. The addition of cooperation among predators can generate a pair of new solutions, both of them corresponding to partial extinctions of prey. The effect is the same that we showed for one population in Figure 1 but acting on the predator axis. Thus, cooperation among predators introduces a similar effect of facultative predation. We tested two different values of predators cooperation parameter $b_{22}$ to see its direct influence. Although, in both cases, the cooperative term is greater than predation, i.e., $b_{21}<b_{22}$, we can see that, at lower values of cooperation, almost no effect is notable, but at greater values, two partial extinctions of prey appear, one stable and one unstable, a saddle-node bifurcation. This allows predators to survive without preys when cooperation 


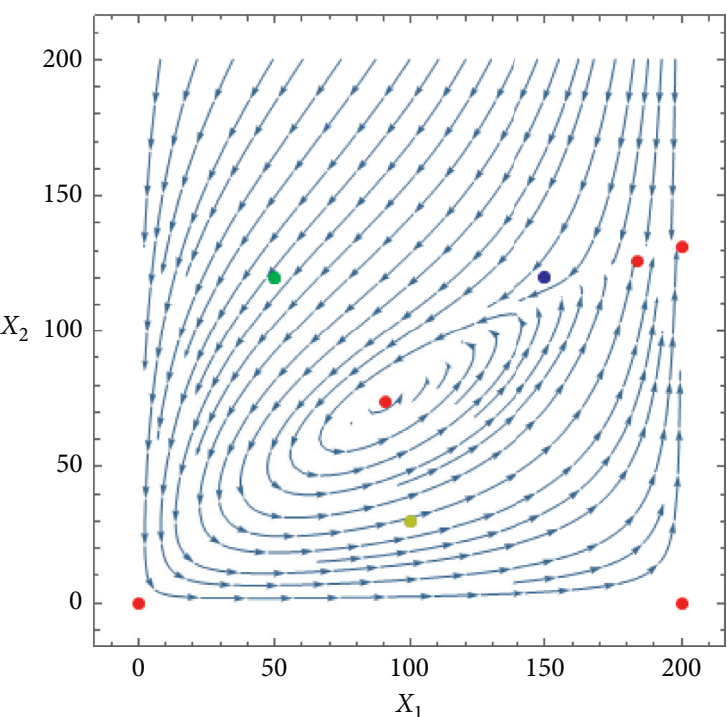

(a)

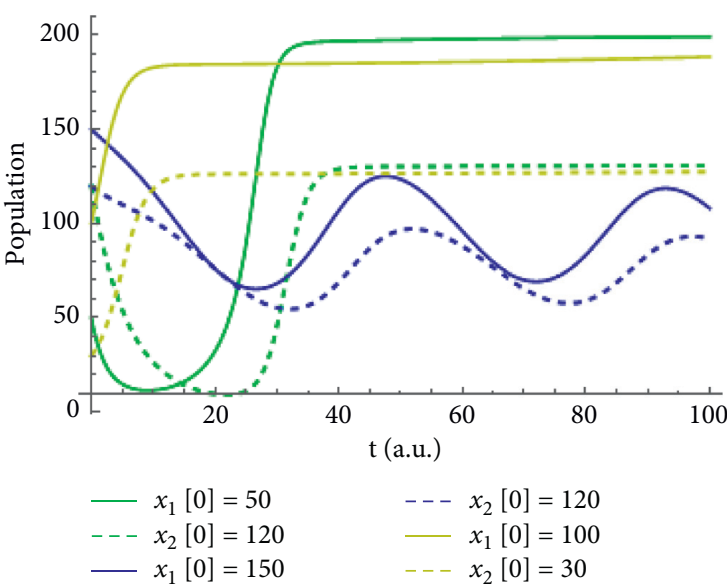

(c)

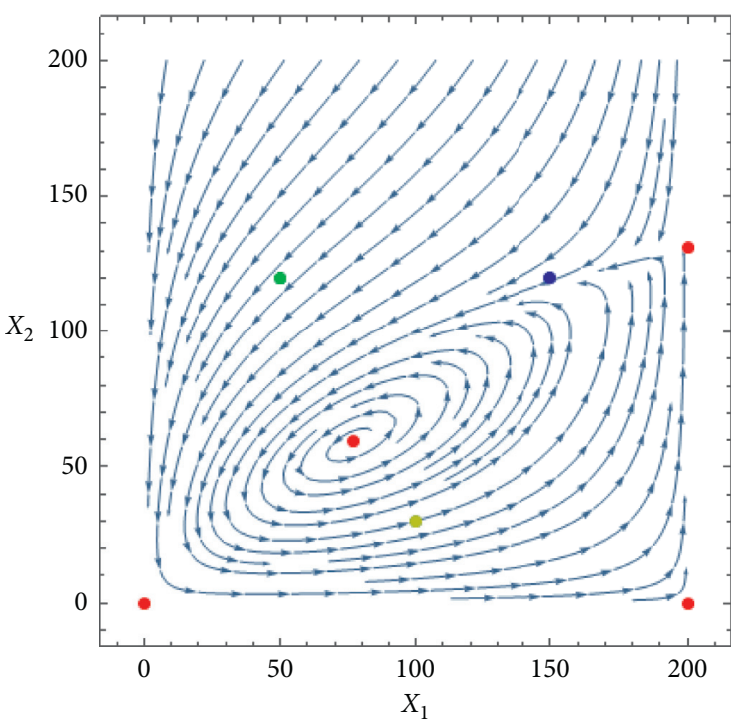

(b)

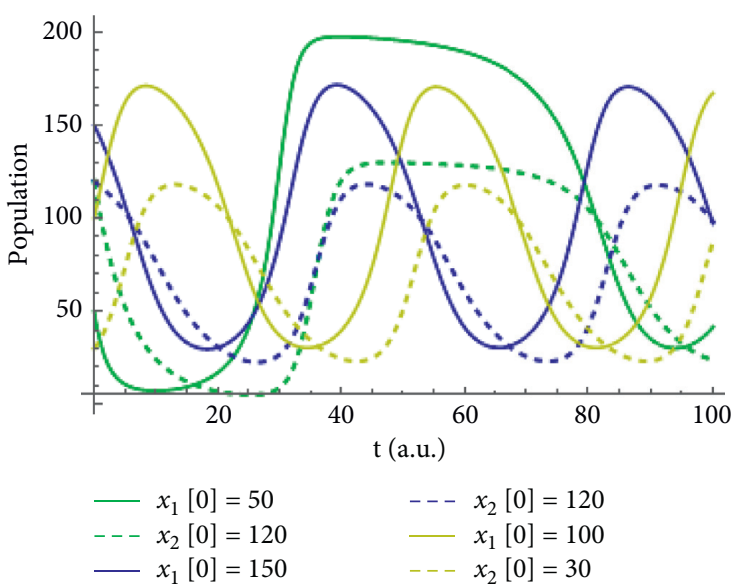

(d)

Figure 5: Phase space and trajectories for two populations involved in a predator-prey interaction. We show here a special case where the coexistence spiral solution diverges and become unstable. When that happens, a limit cycle appears. To the left, we have a case with a smaller predation, i.e., $b_{12}=-0.006435$ than to the right, where $b_{12}=-0.0072$. In both cases, $b_{11}=b_{21}=0.0036$, which means that both populations benefit the same from population $X_{1}$, but the predatory effects of $X_{2}$ on $X_{1}$ are stronger on the right. For greater cooperation values, the intermediate solution might even disappear, as it is shown on the right. The green, blue, and yellow dots in the phase space mark the initial conditions of the simulations located below. Here, $r_{1}=0.15$ and $r_{2}=-0.15$.

reaches a certain limit. In Figure 6(a), we have the case in which cooperation is weaker, and in Figure 6(b), the case in which is mildly stronger. The coexistence located at the carrying capacity of the prey remains unstable.

3.2. Competition. In the case of competition, the principle of competitive exclusion stands that the stable solution is the partial extinction but, if interaction parameters are weak, another feasible stable solution is a coexistence point [17]. However, by including intraspecific interactions, the coexistence could become stable for higher or lower values of the interspecific interaction parameters. For a range of positive intraspecific parameters, partial extinctions and the total carrying capacity could be stable at the same time. Adding a positive intraspecific interaction term (cooperation) in one species may induce a new saddle point, defining two basins, one towards partial extinction of this species and the other one to the system carrying capacity. When cooperation occurs in both species, these two saddle points and the origin define a central attraction basin towards the system carrying capacity; meanwhile, outside this basin, the system evolves towards one species extinction, as per the principle of competitive exclusion (see Figure 7(a)). When we have negative intraspecific parameters, the carrying capacity becomes unstable, and the only stable solutions are the partial extinctions; however, due to the intraspecific interaction, these points occur at a population below its carrying capacity. Both effects can be seen as consequences of intraspecific cooperation and competition in the same way as for one population in Figure 1. Cooperation induces new 


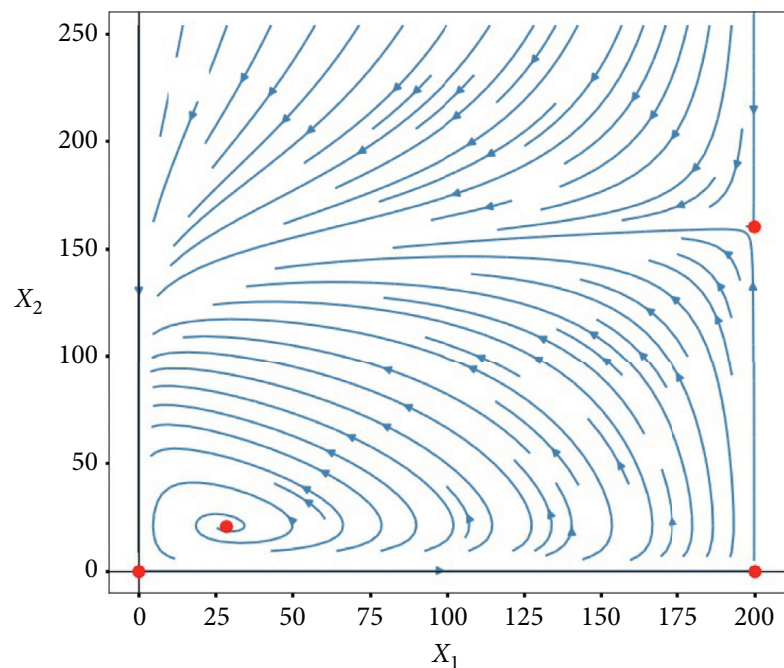

(a)

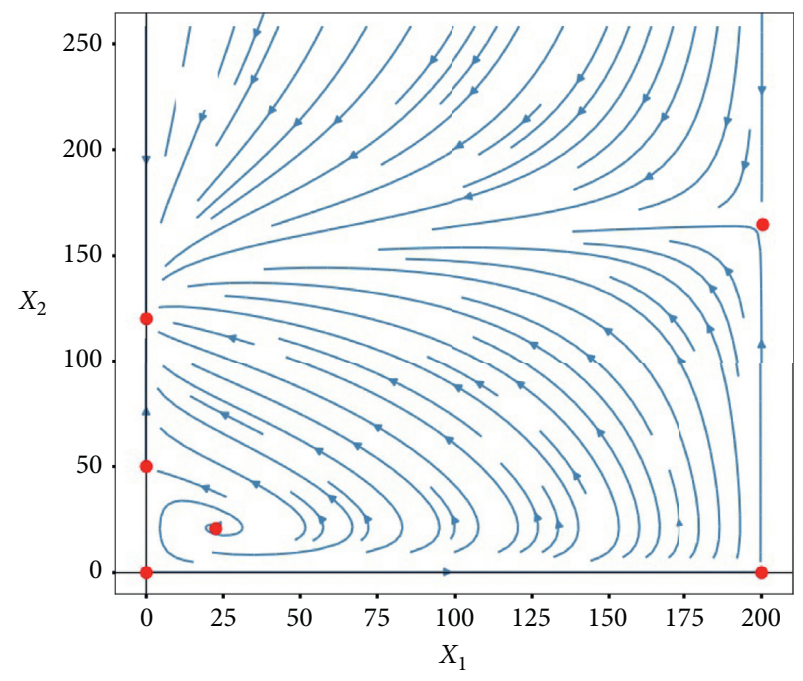

(b)

Figure 6: Phase space and trajectories for two populations involved in an antagonist interaction. To the left, we have a case with a lower $b_{22}=0.004$ than to the right, $b_{22}=0.005$. Cooperation among predators allows two new partial extinctions of prey, one stable and one unstable, in the same way in Figure 1 but on the predators axis. The coexistence located at the carrying capacity of the prey remains unstable. Here, $r_{1}=0.15, r_{2}=-0.15, b_{11}=0, b_{12}=-0.0072$, and $b_{21}=0.0036$.

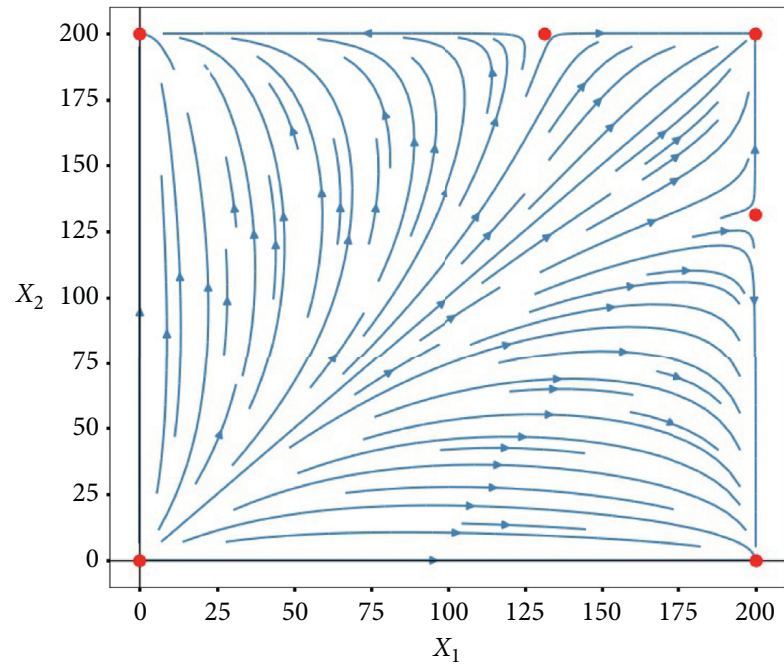

(a)

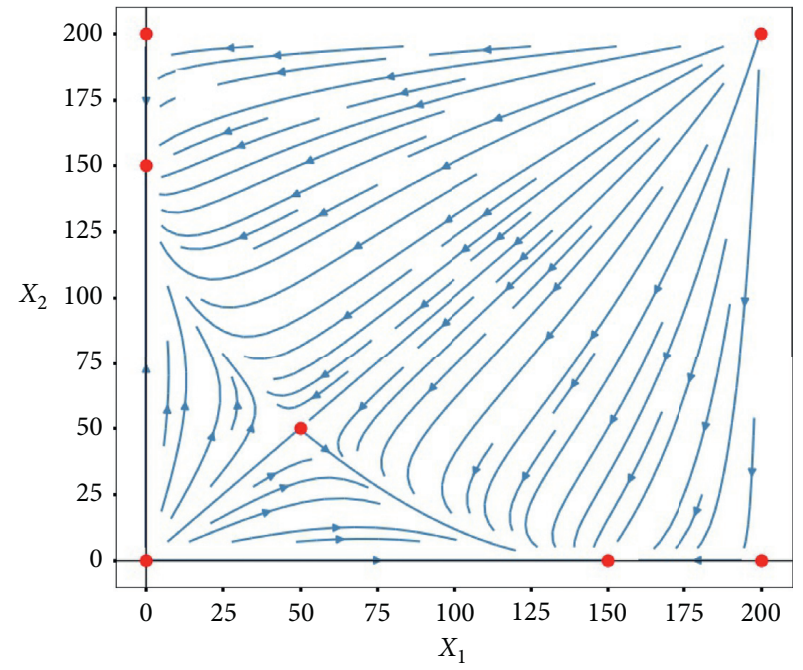

(b)

Figure 7: Phase space and trajectories for two populations involved in competition with positive intraspecific interaction. We used two different combinations of $b_{11}=b_{22}$ to see the influence of intraspecific cooperation and competition. (a) $b_{11}=b_{22}=0.0019$, and we have the case in which both populations are cooperative and two new solutions appear together with a basin towards the carrying capacity of the system. (b) $b_{11}=b_{22}=-0.001$, and we have the case in which both are competitive. Noting that when both populations are cooperative, partial carrying capacities appear and they are both unstable. And when both populations are competitive, partial extinctions appear instead although stable and below the carrying capacities. Here, $r_{1}=r_{2}=0.15$ and $b_{12}=b_{21}=-0.002$.

solutions as partial carrying capacities and intraspecific competition as partial extinctions.

3.3. Mutualism. The logistic-mutualistic model exhibits, in addition to the total and partial extinctions, two feasible finite solutions (5): the larger one corresponds to the case where both populations reach their carrying capacities, and the lower one is a saddle point that allows us to define a survival watershed. By adding intraspecific interactions, new partial extinctions and carrying capacities could appear.

3.3.1. Obligate-Obligate Mutualism. For the sake of simplicity, we only expose the case of equal sign in the parameters for both species, i.e., $r_{1}, r_{2}<0$ and $b_{12}, b_{21}>0$. In Figure 8 , we show the phase space for two populations involved in a mutual obligatory mutualism with two 


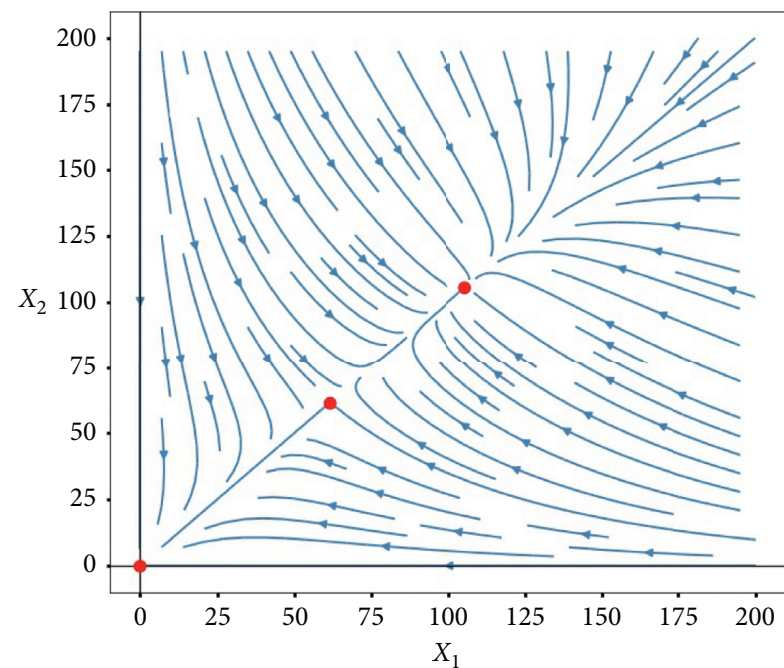

(a)

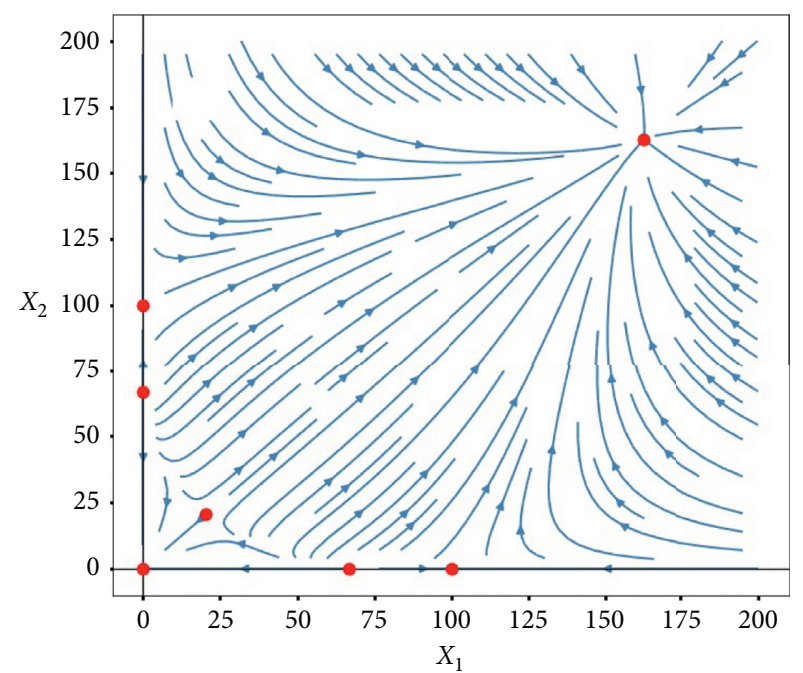

(b)

Figure 8: Obligate-obligate mutualism with cooperation in two populations: (a) we have the case where $b_{11}=b_{22}=0.0001$, which means that intraspecific cooperation is lower than mutualism; (b) we have $b_{11}=b_{22}=0.0045$, which means that both intraspecific cooperation and mutualism weight the same. Here, $r_{1}=r_{2}=-0.15$ and $b_{12}=b_{21}=0.0045$.

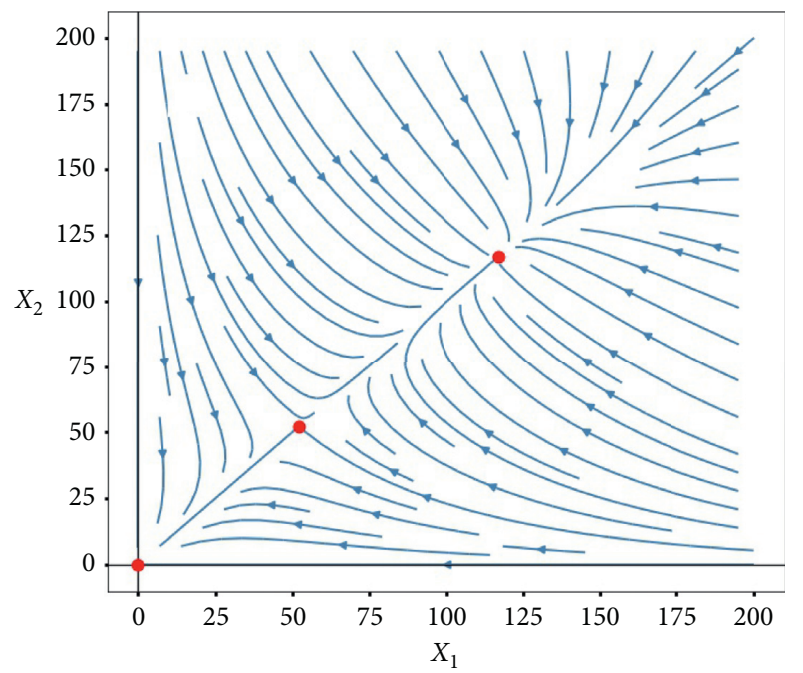

(a)

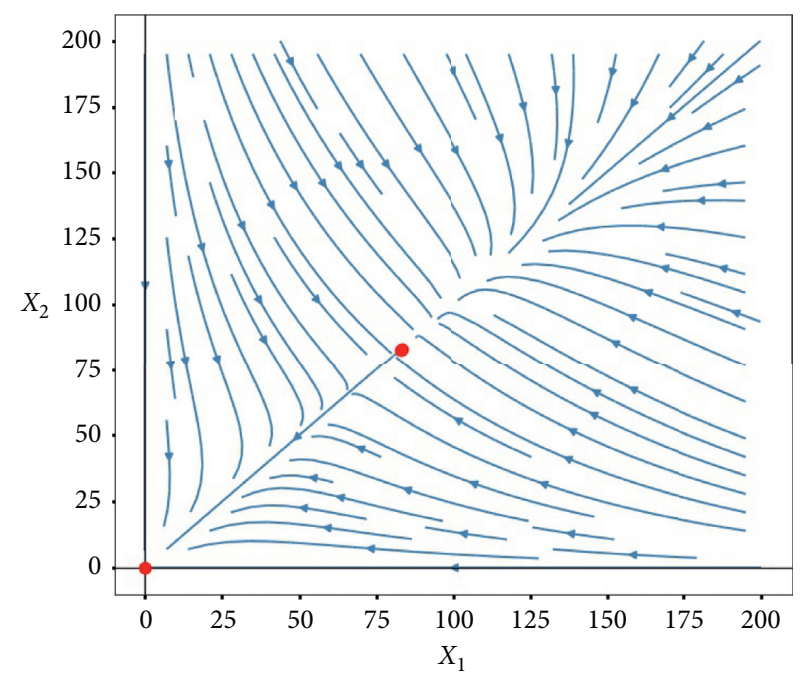

(b)

FIgURE 9: Obligate-obligate mutualism with competition in two populations: (a) we have the case where $b_{11}=b_{22}=-0.0001$, which means that intraspecific competition is lower than mutualism; (b) we have $b_{11}=b_{22}=-0.00062868$, which means that intraspecific competition has stronger effects than mutualism. Here, $r_{1}=r_{2}=-0.15$ and $b_{12}=b_{21}=0.005$.

different values of the cooperation coefficients, $b_{i i}$. In Figure 8(a), with weak cooperation, the phase space exhibits two free-equilibrium points: the stable carrying capacity and a saddle point defining a survival watershed, as in [5]. However, with strong intraspecific interaction (Figure 8(b)), four new unstable solutions can appear: two saddle points and two unstable fixed nodes, corresponding to partial extinctions. As in the case of one population (see Figure 1), the new saddle points are the thresholds. Whenever a population is higher than this threshold, it will never go extinct. The total extinction basin is limited by the curve passing through the nontrivial saddle point and these new unstable fixed nodes.

On the contrary, when mutualistic species exhibits negative intraspecific interactions, as in Figure 9, the stable carrying capacity moves towards the saddle point (Figure 9(a)). And eventually, when this negative term is high enough, these two solutions collide and total extinction remains as the exclusive stable stationary solution (Figure 9(b)).

In the case of one cooperative population and one competitive population, the system exhibits this asymmetry; again, a new saddle point in the cooperative population axis sets a survival threshold. Above it, the system always evolves 


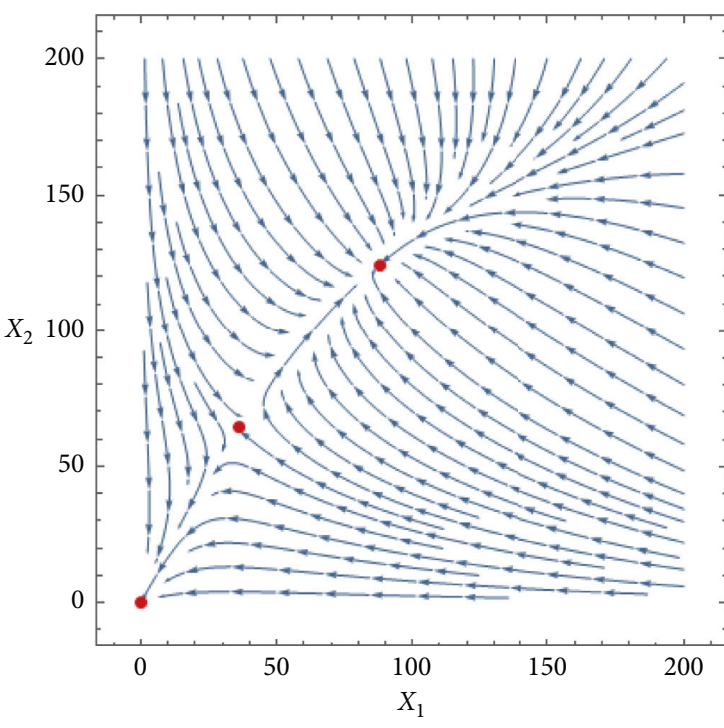

(a)

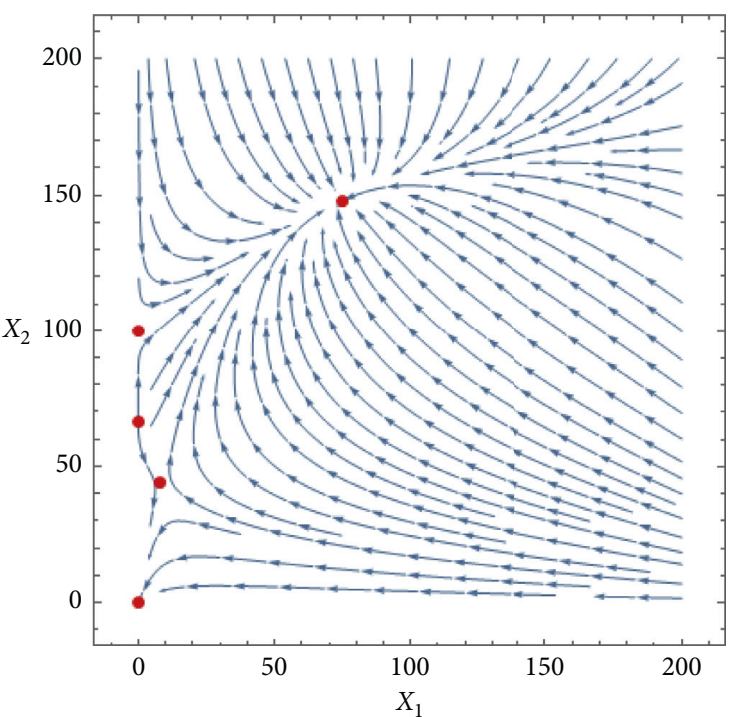

(b)

Figure 10: Obligate-obligate mutualism with positive and negative intraspecific interaction: (a) we have the case where $b_{11}=-0.002$ and $b_{22}=0.002$, which means that intraspecific competition of $X_{1}$ is the same that intraspecific cooperation of $X_{2}$ and both interactions weight lower than mutualism; (b) we have $b_{11}=-0.0045$ and $b_{22}=0.0045$, which means that intraspecific competition of $X_{1}$ weights the same than mutualism and intraspecific cooperation of $X_{2}$. Here, $r_{1}=r_{2}=-0.15$ and $b_{12}=b_{21}=0.0045$.

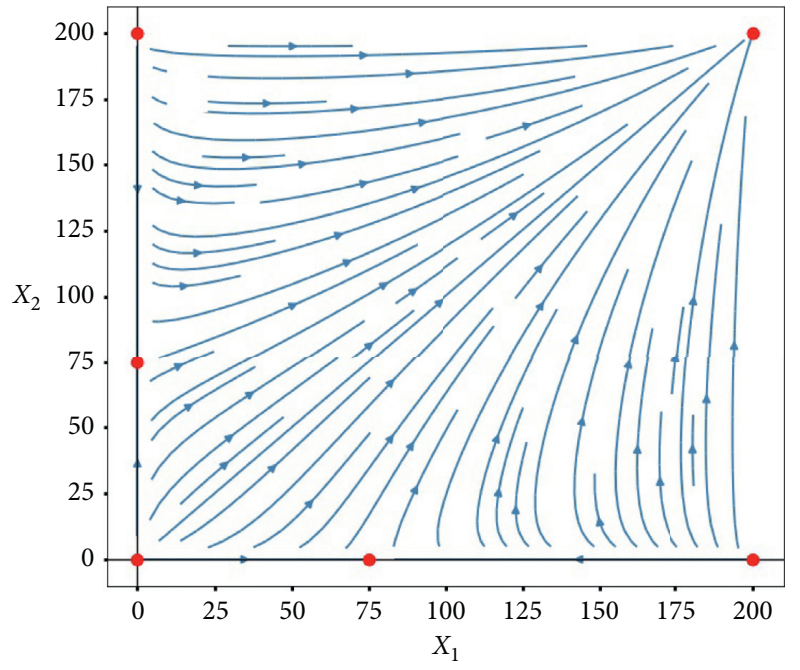

(a)

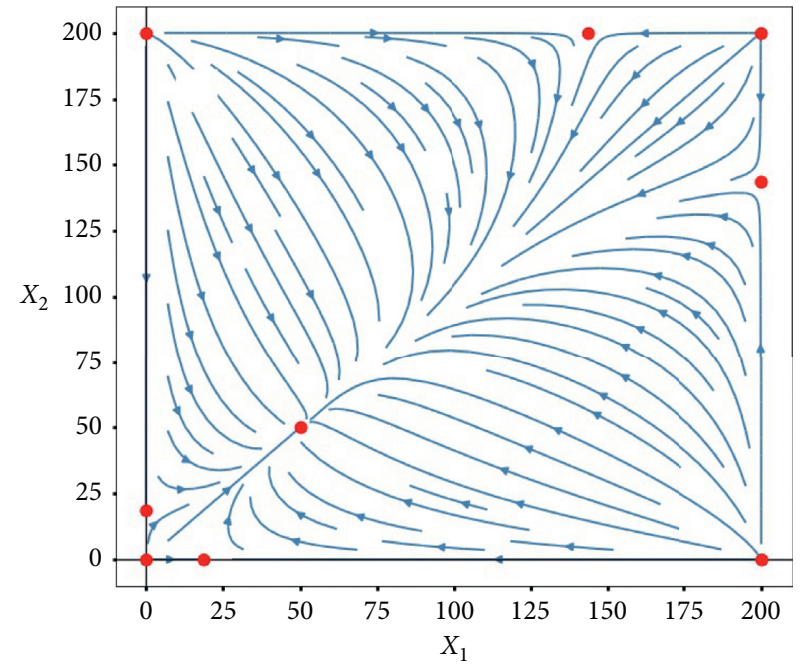

(b)

Figure 11: Facultative-facultative mutualism with negative intraspecific interaction: (a) we have the case where $b_{11}=b_{22}=-0.002$, which means that intraspecific competition is weaker than mutualism; (b) we have $b_{11}=b_{22}=-0.008$, which means that intraspecific competition is stronger than mutualism. Here, $r_{1}=r_{2}=0.15$ and $b_{12}=b_{21}=0.005$.

towards the coexistence solution and will never go extinct, and it is shown in Figure 10.

3.3.2. Facultative-Facultative Mutualism. When both growth rates, $r_{1}$ and $r_{2}$, are positive, total extinction is an unstable solution and the carrying capacity is stable (Figure 11(a)). However, when both populations exhibit negative intraspecific interactions, the maximum system carrying capacity may become unstable and a new stable finite solution emerges at lower populations (Figure 11(b)), as one expects following the one population solution with intraspecific competition (see Figure 1). In Figure 11(a), the intraspecific interaction generates four partial extinctions as unstable stationary solutions (two saddle points and two unstable nodes). In Figure 11(b), with higher negative intraspecific interaction, two extra solutions appear as partial carrying capacities, and the total carrying becomes unstable. In this case, the system exhibits 9 positive stationary 


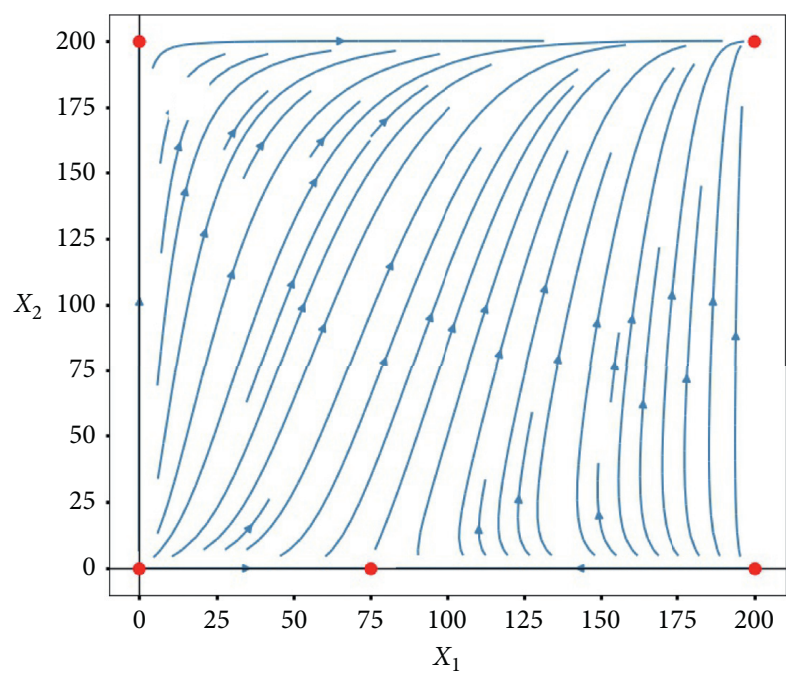

(a)

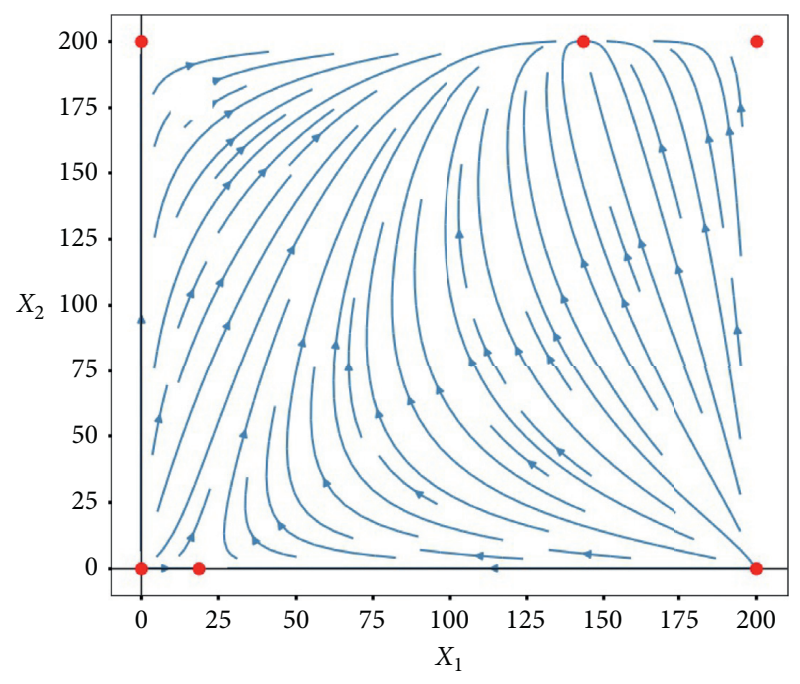

(b)

Figure 12: Facultative-facultative mutualism with intraspecific competition and cooperation: (a) we have the case where $b_{11}=-0.002$ and $b_{22}=0.008$; (b) we have $b_{11}=-0.008$ and $b_{22}=0.002$. Here, $r_{1}=r_{2}=0.15$ and $b_{12}=b_{21}=0.005$.

solutions: four saddle points, four unstable points, and only one stable solution.

In the case of facultative mutualism with different intraspecific interactions, one of them is beneficial and the other one is harmful; the carrying capacity could be reduced for populations with negative intraspecific interaction, while its partner, with positive intraspecific interaction, will grow until reaching its own saturation. Figure 12 depicts this scenario. In Figure 12(a), competition is weaker than cooperation, and the total carrying capacity is the stable stationary solution. In Figure 12(b), competition is stronger than cooperation, and the total carrying capacity becomes unstable. As before, competition only generates unstable a partial extinction, while cooperation pushes the coexistence solution into a transcritical bifurcation.

\section{Conclusions}

In the title of the paper, we ask how simple a population dynamics model should be. To address the discussion, we have introduced the intraspecific interactions in the [5] model using their same philosophy to include new terms. These appear in the first term of the interaction, representing the effective growth rate, and in the logistic brake to balance the first term. With respect to the previous model, this modification introduces two new terms: $b_{i i} X_{i}^{2}$ and $-c_{i} b_{i i} X_{i}^{3}$, regarding the intraspecific interactions. Furthermore, we have generalized the model allowing the parameters that define the interactions, $b_{i j}$, to be positive or negative.

In our opinion, the ecological reason to introduce different intraspecific interactions is supported by observations; cooperative and competitive intraspecific interactions are widely known in a wide variety of ecological systems, from social insects to microbial communities. They have been overseen by population dynamics modelling, which mainly focused on interactions with the environment or interspecific interactions (see, for example, the historical sequence developed in [18]).

Furthermore, the cubic term offers an interesting behaviour from the mathematical point of view. As AlAdwani and Saavedra [11] explain that new high-order terms can introduce new free-equilibrium solutions, but it is necessary that these solutions will be feasible, and of course, with a clear ecological meaning. In this way, several authors have used high-order interactions to improve the stability or diversity of ecological models. For example, Letten and Stouffer [9] show the advantages of the high-order terms, introducing nonadditive density-dependent effects; the authors study the influence of the high-order interactions in the competitive communities. Or Grilli et al. [19] show how the high-order interactions increase the stability of the systems. In our model, the term $-c_{i} b_{i i} X_{i}^{3}$ introduces 2 new free-equilibrium solutions (see Supplementary Material (available here)) that in our opinion can explain ecological situations that were not well explained before with the population dynamics equations.

Delving into the idea of high-order interactions, Bairey et al. [10] introduce 3-way or 4-way terms, overcoming the pairwise interactions. These terms are intended to simulate the effect that interactions between species are modulated by one or more species. This idea is inspiring, but we believe that simpler models like ours that use polynomial terms and pairwise interaction can still explain many ecological landscapes. Every time that we increase the order of a new term, it is more difficult to define it and their corresponding parameters in the field.

We would like to highlight that the inclusion of the intraspecific terms shows new solutions that could represent more complex ecological landscapes. For example, the case of predator-prey system with positive intraspecific term in the preys exhibits a new solution with a steady state at large populations. This solution could represent the way herds act as a defensive mechanism for preys [20,21]. Also, large herds 
of zebras or wildebeest seem to be stable in time; in [22], the authors presented data of the Kruger National Park, in South Africa, that showed a stable and increasing population of zebras and wildebeest (more than 10,000 individuals) over a period of twenty years, with more or less stable population of lions (around 400 individuals). Or the effects of intraspecific competition can act as a regulatory mechanism. Polis [23] showed that intraspecific predation acts in a reinforced way: higher populations decrease the resources available for individuals, reducing their growing rates and promoting smaller and weaker individuals, those are more easily killed or eaten, which increases the per capita food level, both by reducing the population and by satiating the cannibalists.

The main advantage of this general model (equation (3)) is that it can be used to describe any ecological regime and that it carries its own saturation mechanism that avoids the "orgy of mutual benefaction" of [24]. Stucchi [25] showed using a simplified generalized model, studying a nursery pollination system, and modelling all the interspecific interactions with the same functional. This allowed a clear interpretation of the parameters of the whole system and an unambiguous way to compare them. Furthermore, Stucchi $t$ al. [26] showed that intraspecific interactions in a predatorprey system might lead to diffusion-driven instabilities.

Finally, we would like to venture to discuss some more speculative ideas. Nowadays, there are some attempts to model transitions from antagonistic to mutualistic interspecific relationships, limited by the fact that they deal with different mathematical functionals for mutualism and antagonism [27-29]. These models include changes that arise continually from one regime to another, but treating the transition only in a descriptive way. In addition, adaptive changes are modelled through parameter changing systems, where parameters have their own dynamic equations, but these models are still limited to specific ecological regimes, either antagonistic or mutualistic [30-32]. However, if one may adequately define the dynamics of the parameters in a general model of ecological interactions, it may reflect a deeper view of nature, where ecology meets evolution. Thus, by including evolutionary changes in our model, one may be capable of modelling transitions due to mutations and natural selection, which is surely the way how transitions on ecological regimes occur in nature.

\section{Data Availability}

This is a theoretical study, and we do not have experimental data.

\section{Conflicts of Interest}

The authors declare that they have no conflicts of interest.

\section{Authors' Contributions}

All authors contributed equally to the study.

\section{Acknowledgments}

This work was supported by the Ministry of Education, Culture, and Sport of Spain (PGC2018-093854-B-100).

\section{Supplementary Materials}

Quantifying the effect of the intraspecific term. The Jacobian matrix for $N$ species. (Supplementary Materials)

\section{References}

[1] A. J. Lotka, Elements of Physical Biology, Williams and Wilkins Company, Baltimore, MD, USA, 1925.

[2] V. Volterra, "Fluctuations in the abundance of a species considered mathematically," Nature, vol. 118, no. 2972, pp. 558-560, 1926.

[3] J. Bascompte, "Disentangling the web of life," Science, vol. 325, no. 5939, pp. 416-419, 2009.

[4] J. Bascompte, "Mutualistic networks," Frontiers in Ecology and the Environment, vol. 7, no. 8, pp. 429-436, 2009.

[5] J. García-Algarra, J. Galeano, J. M. Pastor, J. M. Iriondo, and J. J. Ramasco, "Rethinking the logistic approach for population dynamics of mutualistic interactions," Journal of Theoretical Biology, vol. 363, pp. 332-343, 2014.

[6] A. M. Dean, "A simple model of mutualism," The American Naturalist, vol. 121, no. 3, pp. 409-417, 1983.

[7] D. H. Wright, "A simple, stable model of mutualism incorporating handling time," The American Naturalist, vol. 134, no. 4, pp. 664-667, 1989.

[8] U. Bastolla, M. A. Fortuna, A. Pascual-García, A. Ferrera, B. Luque, and J. Bascompte, "The architecture of mutualistic networks minimizes competition and increases biodiversity," Nature, vol. 458, no. 7241, pp. 1018-1020, 2009.

[9] A. D. Letten and D. B. Stouffer, "The mechanistic basis for higher-order interactions and non-additivity in competitive communities," Ecology Letters, vol. 22, no. 3, pp. 423-436, 2019.

[10] E. Bairey, E. D. Kelsic, and R. Kishony, "High-order species interactions shape ecosystem diversity," Nature Communications, vol. 7, no. 1, pp. 1-37, 2016.

[11] M. AlAdwani and S. Saavedra, "Is the addition of higher-order interactions in ecological models increasing the understanding of ecological dynamics?" Mathematical Biosciences, vol. 315, Article ID 108222, 2019.

[12] P. F. Verhulst, "Notice sur la loi que la population suit dans son accroissement," Correspondance Mathematique et Physique, vol. 10, pp. 113-117, 1838.

[13] E. O. Wilson, The Insect Societies, Belknap Press, Cambridge, MA, USA, 1971.

[14] B. Stadler and A. F. G. Dixon, "Ecology and evolution of aphid-ant interactions," Annual Review of Ecology, Evolution, and Systematics, vol. 36, no. 1, pp. 345-372, 2005.

[15] M. Stucchi and J. Figueroa, "La avifauna de las islas Lobos de Afuera y algunos alcances sobre su biodiversidad," Asociacion Ucumari. Lima, Perú, Reporte de Investigacion N 2, 2006.

[16] W. C. Allee, Animal Aggregations: A Study in General Sociology, University of Chicago Press, Chicago, IL, USA, 1931.

[17] J. D. Murray, Mathematical Biology I. An Introduction, Springer, Berlin, Germany, 1993.

[18] P. Turchin, Complex Population Dynamics: A Theoretical/ empirical Synthesis (MPB-35), Princeton University Press, Princeton, NJ, USA, 2003.

[19] J. Grilli, G. Barabás, M. J. Michalska-Smith, and S. Allesina, "Higher-order interactions stabilize dynamics in competitive network models," Nature, vol. 548, no. 7666, pp. 210-213, 2017.

[20] D. I. Rubenstein, On Predation, Competition, and the Advantages of Group Living, 205-231, Springer US, Boston, MA, USA, 1978. 
[21] J. Berger, "“Predator harassment" as a defensive strategy in ungulates," American Midland Naturalist, vol. 102, no. 1, pp. 197-199, 1979.

[22] T. H. Fay and C. Greeff, "Lion, wildebeest and zebra: a predator-prey model," Ecological Modelling, vol. 196, no. 1-2, pp. 237-244, 2006.

[23] G. A. Polis, "The evolution and dynamics of intraspecific predation," Annual Review of Ecology and Systematics, vol. 12, no. 1, pp. 225-251, 1981.

[24] R. M. May, "Models for two interacting populations," in Theoretical Ecology: Principles and Applications, pp. 78-104, Oxford University Press, Oxford, UK, 1981.

[25] L. Stucchi, L. Giménez-Benavides, and J. Galeano, "The role of parasitoids in a nursery-pollinator system: a population dynamics model," Ecological Modelling, vol. 396, pp. 50-58, 2019.

[26] L. Stucchi, J. Galeano, and D. A. Vasquez, "Pattern formation induced by intraspecific interactions in a predator-prey system," Physical Review E, vol. 100, no. 8, 2019.

[27] V. I. Yukalov, E. P. Yukalova, and D. Sornette, "Modeling symbiosis by interactions through species carrying capacities," Physica D: Nonlinear Phenomena, vol. 241, no. 15, pp. 1270-1289, 2012.

[28] C. Neuhauser and J. E. Fargione, "A mutualism-parasitism continuum model and its application to plant-mycorrhizae interactions," Ecological Modelling, vol. 177, no. 3-4, pp. 337-352, 2004.

[29] S. Kéfi, V. Miele, E. A. Wieters, S. A. Navarrete, and E. L. Berlow, "How structured is the entangled bank? The surprisingly simple organization of multiplex ecological networks leads to increased persistence and resilience," PLoS Biology, vol. 14, no. 8, Article ID e1002527, 2016.

[30] U. Dieckmann and R. Law, "The dynamical theory of coevolution: a derivation from stochastic ecological processes," Journal of Mathematical Biology, vol. 34, no. 5-6, pp. 579-612, 1996.

[31] A. White and R. G. Bowers, "Adaptive dynamics of LotkaVolterra systems with trade-offs: the role of interspecific parameter dependence in branching," Mathematical Biosciences, vol. 193, no. 1, pp. 101-117, 2005.

[32] J. N. Holland, D. L. DeAngelis, and S. T. Schultz, "Evolutionary stability of mutualism: interspecific population regulation as an evolutionarily stable strategy," Proceedings of the Royal Society B: Biological Sciences, vol. 271, pp. 1807-1814, 2004. 